\title{
Airway obstruction after biopsy by cervical mediastinoscopy in a patient with a mediastinal mass -A case report-
}

\author{
Yong-Cheol Lee ${ }^{2}$, Sang-Jin Park ${ }^{1}$, and In-seong Kim ${ }^{1}$ \\ Department of Anesthesiology and Pain Medicine, ${ }^{1}$ College of Medicine, Yeungnam University, ${ }^{2}$ School of Medicine, Keimyung \\ University, Daegu, Korea
}

Biopsy, using mediastinoscopy is commonly employed for accurate histologic diagnosis of a mediastinal mass. However, since the mass is not removed during the procedure, it may cause compression of vital structures such as major airways, the heart, the pulmonary artery, and the superior vena cava after surgery. We observed a case of a 66 -year-old man with a mediastinal mass that caused severe airway obstruction during recovery from anesthesia following mediastinoscopic biopsy, probably caused by upper airway edema which seemed to originate from compression of the superior vena cava. Therefore, we suggest that unexpected airway obstruction in a patient with a mediastinal mass can be due to superior vena cava compression. (Korean J Anesthesiol 2012; 63: 65-67)

Key Words: Airway obstruction, Mediastinoscopy, Superior vena cava.

Biopsies, using mediastinoscopy are commonly utilized to decide on the treatment of mediastinal masses. However, since the mass is not removed during the biopsy, it may cause compression of vital structures after the procedure [1]. The superior vena cava has an especially low intravascular pressure and thin vascular walls, so it can be easily affected by pressure from a mediastinal mass. In the case discussed here, an airway obstruction developed from supraglottic edema which seemed to originate from pressure on the superior vena cava after biopsy of a mediastinal lesion by mediastinoscopy. We therefore report on the risk of pressure on the superior vena cava and the appropriate management when a mediastinal mass remains after surgery.

\section{Case Report}

A 66-year-old man visited the hospital with a one month history of chest discomfort and sporadic swelling in the face and arms in the morning. No edema or distention of the neck veins was present on physical examination. Positron emission tomography (PET) revealed a hypermetabolic mass on the right side of the distal trachea. A chest-computed tomography (CT) found a $6.5 \mathrm{~cm}$ mass in the same area that was compressing the superior vena cava (Fig. 1). A biopsy using mediastinoscopy was planned to determine the method of treatment.

Because of possible airway obstruction during anesthesia, preparation was made to perform an immediate anterior

Received: September 27, 2011. Revised: 1st, November 1, 2011; 2nd, November 14, 2011; 3rd, November 20, 2011. Accepted: November $28,2011$. Corresponding author: Sang-Jin Park, M.D., Department of Anesthesiology and Pain Medicine, College of Medicine, Yeungnam University, 317-1, Daemyung-dong, Nam-gu, Daegu 705-717, Korea. Tel: 82-53-620-3365, Fax: 82-53-626-5275, E-mail: apsj0718@naver.com (c) This is an open-access article distributed under the terms of the Creative Commons Attribution Non-Commercial License (http:// creativecommons.org/licenses/by-nc/3.0/), which permits unrestricted non-commercial use, distribution, and reproduction in any medium, provided the original work is properly cited. 


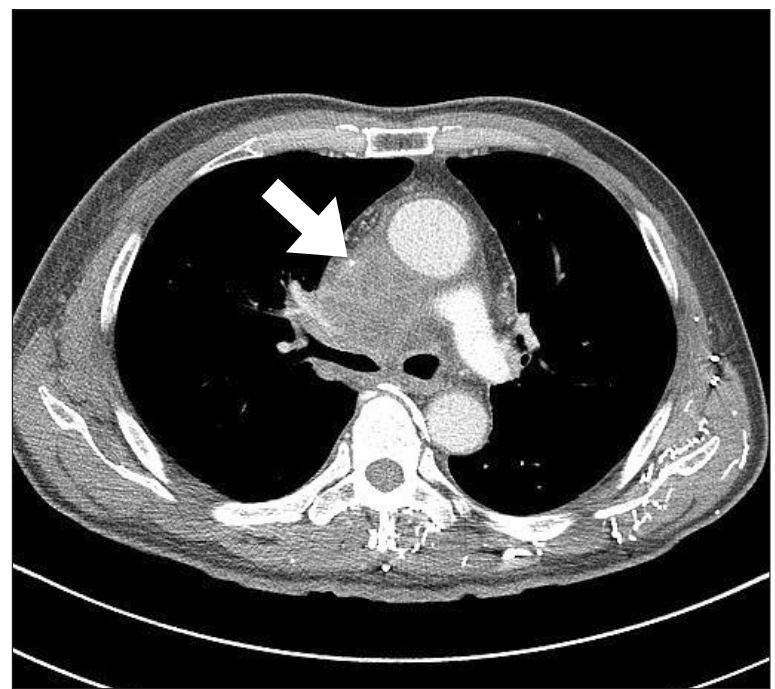

Fig. 1. A chest CT shows that mass is pressing the superior vena cava (arrow).

sternotomy to relieve airway pressure, if necessary. This procedure was explained to the patient and guardian beforehand and their consent was obtained. After arriving in the operating room, the patient had no particular breathing problems in the supine position and no edema was present. Propofol $100 \mathrm{mg}$ was injected for induction of anesthesia. After checking for proper mask ventilation, rocuronium $50 \mathrm{mg}$ was administered. Ventilation was accomplished without any difficulties. During the endotracheal intubation, the patient was graded at Cormack \& Lehane grade II. No peculiarities existed in the oral cavity or glottis area, and intubation $(8.0 \mathrm{~mm})$ was performed easily. To perform the biopsy, the neck was extended from a 10 degree Trendelenburg position, and a mediastinoscope was inserted through the sternal notch. The biopsy was successful. Vital signs were stable throught the procedure, and the length of the operation was 3 hours and 10 minutes.

When the surgical drape was removed, edema and cyanosis were observed in the face. However, when the air in the tracheal tube cuff was removed, leakage of anesthetic gas was found around the tube. As the patient recovered from anesthesia, he breathed normally and raised his hands in response to verbal commands. Therefore, extubation was performed. However, after extubation, the supraclavicular lesion and sternal notch retracted from expansion of the lower thorax and abdomen. No movement occurred in the reservoir bag. The patient's mouth was firmly closed, so an oral airway could not be inserted, and airway obstruction was not relieved even with jaw thrusts. A nasal airway was therefore inserted. When positive ventilation was performed with the facial mask, the airway pressure rose to $30 \mathrm{cmH}_{2} \mathrm{O}$ and the tidal volume was $100-$ $200 \mathrm{ml}$. A few seconds later, blood was expelled through the nares and the pulse oximetry reading was $95 \%$. Propofol 120 $\mathrm{mg}$ and succinylcholine $80 \mathrm{mg}$ were injected and endotracheal intubation was attempted. However, the tongue had swollen, becoming markedly larger than before the anesthesia, making the insertion and control of the laryngoscope difficult, and two of the upper teeth were damaged in the process. In addition, a large amount of blood had pooled in the posterior pharynx, impeding visualization of the epiglottis, and pulse oximetry reading decreased to $85 \%$. Therefore, an oral airway was inserted and manual ventilation was performed. Subsequently, the tidal volume and pulse oximetry reading increased to 300$400 \mathrm{ml}$ and $99 \%$, but the airway pressure remained at $30 \mathrm{mmHg}$ and blood continued to be expelled through the nares. Stridor could be heard on auscultation. As the patient's consciousness returned, he shook his head from side to side and ejected the oral airway, once again making ventilation difficult. At this point, propofol $80 \mathrm{mg}$ and rocuronium $70 \mathrm{mg}$ were administered, and endotracheal intubation was attempted using the Glidescope (glidescope, Verathon Medical $^{\mathrm{TM}}$, Burnaby, Canada). However, controlling the Glidescope and securing a clear view were difficult due to the enlarged tongue and accumulated blood. Therefore, to prepare for tracheostomy, endotracheal intubation was attempted with an intubation fiberscope (Olympus LF-GP, Olympus Medical Systems Corp, Tokyo, Japan). The intubation fiberscope was inserted and blood in the oral cavity was continually suctioned off. The epiglottis could be seen, and was observed to be swollen and extended towards the glottis. The left and right arytenoids also appeared to be joined together due to the edema. When the intubation fiberscope was inserted through the arytenoids, the trachea came into view. Although a slight resistance to the tracheal tube was encountered, mounting it in the trachea was possible. Ventilation was smoothly achieved and arterial blood gas analysis (ABGA) after intubation showed $\mathrm{pH} 7.19, \mathrm{PaO}_{2} 311 \mathrm{mmHg}$, and $\mathrm{PaCO}_{2} 56$ mmHg. Otorhinolaryngological examination revealed, overall edema and continuous bleeding caused by damage to the nasal mucosa. The patient was moved to the intensive care unit (ICU) after intranasal packing and removal of the damaged teeth. In the ICU, controlled ventilation was performed in a 30 degree reverse Trendelenburg position. To remove the edema, furosemide and steroids were administered. Following this treatment, the edema in the patient's face subsided, which allowed for removal of the tracheal tube 4 days after surgery. The patient's breathing and vital signs remained stable. Since there was no reason to suspect a neurological disorder, the patient was moved to the general ward.

\section{Discussion}

Obstruction of the superior vena cava leads to chest pain, 
upper body edema, expansion of neck and thoracic veins, and cyanosis [1-3]. These changes can increase cervical venous pressure by $20-40 \mathrm{mmHg}$, which can cause airway obstruction by nasal and laryngeal edema $[2,4]$. In our case, a chest-CT confirmed that the superior vena cava was compressed by a mediastinal mass. The patient also had chest pain, and sporadic edema in the face and arms before the surgery. This pressure on the superior vena cava and a decline in venous return from the upper body could be exacerbated by the neck extension and Trendelenburg position employed during surgery. Superior vena cava compression could cause the enlarged tongue, as well as the edema present in the epiglottis and arytenoids. Therefore, airway edema due to pressure on the superior vena cava from the mediastinal mass appears to have been the cause of the airway obstruction in the case discussed here. There have also been reports of cerebral edema occurring from a decline in venous return in patients undergoing laparoscopic surgery in the Trendelenburg position [5]. Direct pressure of the mass on the trachea as well as the bronchus is reportedly the main cause of airway obstruction $[1,6]$. However, in our case, the chest CT showed that the mass was located on the right side of the distal trachea, and, since the surgery was conducted in the supine position and proper ventilation was possible after tracheal intubation, the chance of direct airway compression by the mass was low.

When symptoms of airway edema from compression of the superior vena cava are present, increasing venous return by elevating the upper body and administering diuretics and steroids can be useful [2]. Although in this case, the authors had to reintubate because of airway obstruction, no problems were experienced in extubation after diuretics and steroids were administered in a reverse Trendelenburg position. In contrast, the supine position or Trendelenburg position can impair venous return due to pressure on the superior vena cava. Therefore, if the upper body had been elevated during surgery in this case, airway edema could have been prevented. In addition, if airway edema is suspected, a cuff-leak test and a measurement of air-column width in the larynx using ultrasonography can predict airway patency after extubation [7]. However, the cuff-leak test has low reliability [8] and ultrasonography has difficulty evaluating supraglottic lesions, such as those involving the tongue and epiglottis. Therefore, an evaluation of the supraglottic airway with the Glidescope or intubation fiberscope, as well as performance of the cuff-leak test before extubation would have been more useful.

A nasal airway is useful to relieve airway obstruction when it is impossible or inadvisable to use an oropharyngeal airway, but has the drawback of causing frequent nasal bleeding [9]. Moreover, in this case, the neck extension and Trendelenburg position could worsen the venous hypertension via pressure on the superior vena cava. This seemed to cause edema not only in the oral cavity but also in the nasal mucosa, and to heighten the possibility of nasal bleeding. In an examination performed after reinsertion of the tracheal tube, edema in the nasal mucosa was observed. There have also been reports of severe nasal bleeding occurring from a mediastinal mass compressing the superior vena cava [10]. If we had mounted an oral airway or attempted endotracheal intubation instead of nasal airway insertion, we could have more easily relieved the airway obstruction without causing bleeding.

In summary, neck extension and Trendelenburg position can cause decreased venous return due to compression of the superior vena cava by a mediastinal mass, and induce edema in the oral and nasal cavity, with consequent airway obstruction. Therefore, performing surgery with the upper body elevated and conducting a thorough post-anesthetic examination of the airway before extubation is suggested in a patient with a mediastinal mass. In addition, since a high possibility of nasal bleeding from edema in the nasal mucosa exists, caution is advised when using a nasal airway.

\section{References}

1. Chung SY, Han YS, Lee MR, Lee HS, Chung CK, Park DH. Postoperative airway obstruction in a patient with huge mediastinal hemangioam. Korean J Anesthesiol 1992; 25: 1028-33.

2. Wilson LD, Detterbeck FC, Yahalom J. Clinical practice. Superior vena cava syndrome with malignant causes. N Engl J Med 2007; 356: 1862-9.

3. Bagheri R, Rahim M, Rezaeetalab F, Akbari H, Shojaian R. Malignant superior vena cava syndrome: is this a medical emergency? Ann Thorac Cardiovasc Surg 2009; 15: 89-92.

4. Mineo TC, Ambrogi V, Nofroni I, Pistolese C. Mediastinoscopy in superior vena cava obstruction: analysis of 80 consecutive patients. Ann Thorac Surg 1999; 68: 223-6.

5. Pandey R, Garg R, Darlong V, Punj J, Chandralekha, Kumar A. Unpredicted neurological complications after robotic laparoscopic radical cystectomy and ileal conduit formation in steep trendelenburg position: two case reports. Acta Anaesthesiol Belg 2010; 61: 163-6.

6. Mackie AM, Watson CB. Anaesthesia and mediastinal masses. A case report and review of the literature. Anaesthesia 1984; 39: 899903.

7. Ding LW, Wang HC, Wu HD, Chang CJ, Yang PC. Laryngeal ultrasound: a useful method in predicting post-extubation stridor. A pilot study. Eur Respir J 2006; 27: 384-9.

8. Kriner EJ, Shafazand S, Colice GL. The endotracheal tube cuff-leak test as a predictor for postextubation stridor. Respir Care 2005; 50: 1632-8.

9. Roberts $\mathrm{K}$, Whalley H, Bleetman A. The nasopharyngeal airway: dispelling myths and establishing the facts. Emerg Med J 2005; 22: 394-6.

10. Sawka AM, Prakash UB. 59-year-old man with epistaxis, headache, and cough. Mayo Clin Proc 2000; 75: 193-6. 